\title{
Effect of cycling shoe cleat position on biomechanical and physiological responses during cycling and subsequent running parts of a simulated Sprint triathlon: a pilot study
}

\author{
Geoffrey Millour', Loic Janson², Sébastien Duc ${ }^{1}$, Frédéric Puel' \& William Bertucci' \\ 1 Laboratory of Performance, Health, Metrology, and Society (EA 7507), UFR STAPS, University of \\ Reims Champagne-Ardenne, Reims, France \\ 2 French Triathlon Federation, Reims, France
}

* Correspondence: Geoffrey Millour.geoffrey.millour@univ-reims.fr

Received: 17 July 2019; Accepted: 17 April 2020; Published: 30 June 2020

\begin{abstract}
Proper cycling cleat adjustment could improve triathlon performance. Recommendations have included positioning the first metatarsal head above the pedal spindle or slightly forward, but mid-foot cleat positions could be more appropriate in triathlon at constant load due to their impact on the subsequent running performance. We evaluated the impact of antero-posterior cleat position on biomechanical and physiological variables during the cycling and running parts of a simulated Sprint triathlon. Seven participants performed two 32-min cycling tests including 8 sets of $3 \mathrm{~min} 30 \mathrm{~s}$ intervals performed at just below the power output at the first ventilatory threshold interspersed with $30 \mathrm{~s}$ sprints at $>100 \%$ of the maximal aerobic power. The cycling exercises were immediately followed by a maximal running performance of $20 \mathrm{~min}$. The tests were performed with a $5-\mathrm{mm}$ posterior $(\mathrm{PCP})$ and a 5-mm anterior (ACP) first metatarsal cleat position. The PCP decreased the energy cost of running ($5.9 \% ; \mathrm{p}=0.04 ;$ effect size $[\mathrm{ES}]=0.37)$ despite no significant change in cycling power output $(\mathrm{p}=0.17$; $\mathrm{ES}=0.40)$ and running speed $(\mathrm{p}=0.89 ; \mathrm{ES}=0.04)$. In addition, the PCP resulted in a lower recruitment of soleus during sub-maximal cycling intensity $(-7.0 \% ; \mathrm{p}=0.04$; ES $=0.88)$ and of gastrocnemius medialis during the running part of the test $(-25 \% ; \mathrm{p}=0.04 ; \mathrm{ES}=1.05)$. Therefore, the PCP could be more suitable in triathlon by being more economical for subsequent running, due to a lower recruitment of calf-muscles during the sub-maximal cycling and the running part.
\end{abstract}

Keywords: Antero-posterior shoe-cleat position, oxygen consumption, EMG, pacing

\section{Introduction}

A triathlon is a multi-task endurance sport, which combines successive swimming, cycling, and running parts (Hausswirth et al. 1996). Triathlon races are classified into four main categories according to the length of the component parts: Sprint $(0.75 \mathrm{~km}$ swimming, 20 $\mathrm{km}$ cycling, and $5 \mathrm{~km}$ running), Olympic ( $1.5 \mathrm{~km}$ swimming, $40 \mathrm{~km}$ cycling, and $10 \mathrm{~km}$ running), Half-Ironman ( 1.9 km swimming, 90 km cycling, and $21 \mathrm{~km}$ running), and Ironman $(3.8 \mathrm{~km}$ swimming, $180 \mathrm{~km}$ cycling, and $42 \mathrm{~km}$ running). The rules of the International Federation of Triathlon are not the same for these four race categories. For example, drafting, which consists of riding behind another triathlete or a group, is authorised only during Sprint and Olympic triathlons. This theoretically allows for saving energy during the cycling leg of the race and creates optimal conditions for the subsequent running performance (Hausswirth et al. 1999; Hausswirth et al. 2001). However, interactions with other triathletes requires producing high 
crank power output (PO) during short periods to follow the others when they accelerate or simply to get a better position in the group (Bernard et al. 2009). In addition, the cycling part of Sprint and Olympic triathlons is generally performed on flat urban tracks including many turns and roundabouts. Two studies reported that triathletes spent between $17 \%$ and $18 \%$ of the cycling time above their maximal aerobic power (MAP) during an Olympic triathlon (Bernard et al. 2009; Etxebarria et al. 2014). Moreover, Etxebarria et al. (2014) noticed that triathletes performed $34 \pm 14$ bursts of $\mathrm{PO}$ above $600 \mathrm{~W}$ during an Olympic triathlon.

Whatever the race category, the overall performance during a triathlon is determined by the ability of the triathlete to do the three disciplines one after the other as faster as possible (Hausswirth and Brisswalter 2008). However, several cycling factors have been identified to play a key role for the subsequent running performance: cycling pacing strategy (Hausswirth et al. 1999; Hausswirth et al. 2001; Hausswirth et al. 2010; Wu et al. 2015), pedalling cadence (Bernard et al. 2003), level of cycling PO (Bernard et al. 2007), cycling position of the triathlete (Garside and Doran 2000), and notably, cleat position (Illes et al. 2010; Paton and Jardine 2012; Viker and Richardson 2013).

Proper adjustment of the cleat position can optimise lower limb kinematics during cycling, which could improve performance (FitzGibbon et al. 2016). It has been empirically established that the first metatarsal head should be positioned directly above the pedal spindle (De Vey Mestdagh 1998; Silberman et al. 2005). Recent studies advise placing the pedal spindle slightly backward, in alignment with the middle of the first and fifth metatarsal heads (FitzGibbon et al. 2016; Burt 2014). This adjustment, also based on empirical knowledge, could improve foot stability, due to a reduction in the lever arm of the foot (pedal axis to ankle joint), and reduce risk of Achilles tendon injury (Burt 2014). Some studies have found no significant impact of very large antero-posterior cleat displacements $(\approx 5 \mathrm{~cm})$ on physiological and performance variables during sub-maximal pedalling (Paton 2009, Van Sickle and Hull 2007). However, no studies have assessed the impact of small changes of cleat position $(<1.5$ $\mathrm{cm})$ at intermittent intensities.

Two studies have investigated the effects of a mid-foot cleat position on biomechanical and physiological variables during the cycling and running parts of a simulated Sprint triathlon (Paton and Jardine, 2012; Viker and Richardson 2013). Paton and Jardine (2012) showed that a mid-foot cleat position improves the subsequent performance of a $5.5 \mathrm{~km}$ time trial running exercise after $30 \mathrm{~min}$ of cycling at $65 \%$ of the MAP. It has been suggested that this advantage could be the result of a decrease of fatigue in the plantar flexor muscles during the cycling part as no reduction in oxygen consumption had been observed. This hypothesis seems to be supported by Litzenberger et al. (2008) and McDaniel et al. (2013), who found that a posterior cleat positioning decreases calf-muscle activity by reducing the lever arm of the foot and, consequently, ankle joint torque. Litzenberger et al. (2008) assume that the less strained calf-muscles could be advantageous for the subsequent running part of a triathlon. However, contrary to Paton and Jardine (2012), Viker and Richardson (2013) found no significant performance improvement during a $5 \mathrm{~km}$ time-trial running exercise if the 30-min cycling part was performed at a non-constant PO (between $50 \%$ and $90 \%$ of the MAP). Nevertheless, the pacing strategy during the running test was significantly modified as the first kilometre was performed more slowly with the mid-foot cleat position compared to using the first metatarsal cleat position.

Although these two previous studies provide some interesting findings, supra-maximal intensities (i.e. above $100 \%$ of the MAP) encountered during the cycling part of Sprint and Olympic triathlons, resulting from interactions with other triathletes and urban track characteristics (Bernard et al. 2009), were not included in the cycling exercise. Moreover, the mid-foot cleat position corresponded to a 
very large posterior cleat displacement $(\approx 5 \mathrm{~cm})$ incompatible with the possible settings of the usual cycling shoes which generally offer a small fore-aft adjustment range (10 to $15 \mathrm{~mm}$ ) or about $20 \mathrm{~mm}$ for high-end shoes (e.g. S-Works EXOS [Specialized Bicycle Components, Inc., Morgan Hill, CA USA] and S-Phyre [Shimano Inc., Sakai City, Osaka, Japan]).

The purpose of the current study was to determine the impact of small shoe-cleat displacements $(1 \mathrm{~cm})$ on biomechanical and physiological variables during the cycling and running parts of a simulated Sprint triathlon which included supra-maximal cycling intensities. The hypothesis was that the posterior cleat position during the cycling part of a Sprint triathlon, characterised by large intensity variations, modifies lower limb muscle activity and increases subsequent running performance without changing cycling performance.

\section{Methods}

\section{Participants}

A small sample of seven participants (five males and two females) were recruited for this preliminary study (mean \pm standard deviation [SD]: $22 \pm 11$ years of age; $1.73 \pm 0.09 \mathrm{~m} ; 60.8 \pm$ $7.7 \mathrm{~kg}$ ). All participants had at least three years of triathlon experience and had trained between 5 and 15 hours per week (taking into account swimming, cycling, and running) in the six months prior to the experiment. In addition, they reported no pain or injuries in the previous year. All participants and the parents of the minor participants were informed of the risks and benefits of the study and they provided written informed consent. The study was approved by the University of Reims Champagne-Ardenne's biomedical research ethics committee in accordance with the Declaration of Helsinki and was conducted in agreement with the ethical guidelines (Harris and Atkinson 2011).

\section{Protocol}

The participants performed three tests separated by 3 days: a graded maximal cycling test and two experimental tests which simulated the cycling to running legs of a Sprint triathlon (Figure 1). They were instructed not to perform high intensity training or a long training session on the day prior to testing.

During the first visit in the laboratory, each participant performed a graded maximal cycling test until exhaustion to determine the MAP, maximal oxygen consumption (VO2max), and power output at first ventilatory threshold (POVT). During a draft-legal triathlon, triathletes spend about $55 \%$ of the time below the POVT (Bernard et al. 2009). To identify the POVT, we used the ventilatory equivalent method which consists of determining the $\mathrm{PO}$ that corresponds with a systematic increase in the ventilatory equivalent of oxygen (O2) without a concomitant increase in the ventilatory equivalent of carbon dioxide (CO2) (Amann et al. 2004). The tests were performed on a cycle-ergometer (Wattbike Ltd., Nottingham, UK). The usual bike position of each participant (e.g. saddle height, saddle fore-aft, and handlebar position) was reproduced on the cycle-ergometer. After $10 \mathrm{~min}$ of warming-up with unrestricted $\mathrm{PO}$, the graded maximal test started at $100 \mathrm{~W}$ for the males and at $70 \mathrm{~W}$ for the females for $2 \mathrm{~min}$. The PO was then increased every 2 min by $20 \mathrm{~W}$ until exhaustion of each participant. They were required to pedal continuously at $80 \pm 5 \mathrm{rpm}$. The criteria of exhaustion were (a) the voluntary cessation, (b) when they were not able to maintain the required $\mathrm{PO}$, or (c) when they were not able to maintain a pedalling cadence superior to $75 \mathrm{rpm}$. After 15 min of passive recovery, the participants performed $10 \mathrm{~min}$ of habituation running on a motorised treadmill (T90, Tunturi New Fitness BV, Almere, Netherlands) inclined to $1 \%$ to simulate outdoor running conditions (Jones and Doust 1996) at a moderate speed $(10 \mathrm{~km} / \mathrm{h})$.

During the second and the third visits, each participant performed an experimental test consisting of successively cycling for $32 \mathrm{~min}$ on the cycle-ergometer and then running for $20 \mathrm{~min}$ on the treadmill, using two cleat positions in random order. The centre of the cleat was positioned $5 \mathrm{~mm}$ in front of the first metatarsal head (anterior cleat position: $\mathrm{ACP}$ ) or $5 \mathrm{~mm}$ 
behind the first metatarsal head (posterior cleat position: PCP) (Figure 2). The participants were allowed to pedal with their own bike shoes and clipless pedals. However, if it was impossible to set the fore-aft cleat position under their own shoes due to a short adjustment range (10-20 $\mathrm{mm})$, they were required to use other laboratory shoes (B'TWIN, Decathlon SA, Villeneuved'Ascq, France) with SPD cleats (Shimano Inc.). The laboratory shoes were eventually equipped with a custom-made system allowing for an increase in the adjustment range (Figure 3). To compensate for the effective decrease in leg length with the posterior cleat position, the saddle height was positioned $5 \mathrm{~mm}$ lower in this condition (Paton and Jardine 2012). In addition, saddle height was positioned $5 \mathrm{~mm}$ higher when cyclists used the custom-made system for the cleat placement. To simulate intermittent $\mathrm{PO}$ encountered during a Sprint triathlon race, due to interactions with other triathletes and urban track characteristics, the cycling part was divided into 8 intervals comprising $3 \mathrm{~min}$ and $30 \mathrm{~s}$ performed at just below the POVT (Zone 1; PO < POVT) at $80 \mathrm{rpm}$ followed by $30 \mathrm{~s}$ all-out sprints (Zone 4; $\mathrm{PO}>100 \%$ of the MAP) at a free pedalling cadence. Participants had to produce the highest PO during the overall cycling test by pacing the intensity as best as possible during Zone 1 and by producing the maximal PO during the sprints. The two intensity zones were chosen according to a study by Bernard et al. (2009) which showed that approximately $55 \%$ of total PO produced during a draft-legal triathlon race is performed in Zone 1 , while $17 \%$ is performed in Zone 4 . During the running exercise, the participants had to run the maximum distance over a time trial (TT) test of $20 \mathrm{~min}$ (TT20) on the motorised treadmill inclined to $1 \%$ (Jones and Doust 1996). In order to simulate race conditions encountered during a triathlon, they could increase or decrease the treadmill speed throughout the TT20 test. The participants had only $1 \mathrm{~min}$ to change their shoes between the cycling exercise and the running exercise.

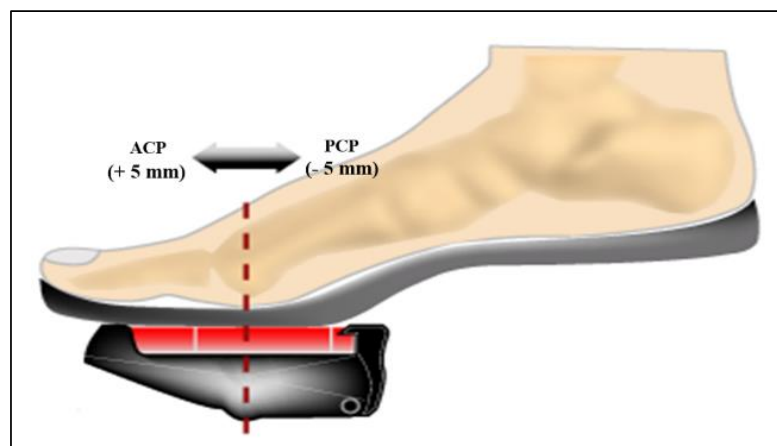

Figure 2: The two cleat positions used during the cycling part of the two experimental tests. The centre of the cleat was positioned $5 \mathrm{~mm}$ in front of the first metatarsal head (Anterior Cleat Position: ACP) or $5 \mathrm{~mm}$ behind the first metatarsal head (Posterior cleat Position: PCP).

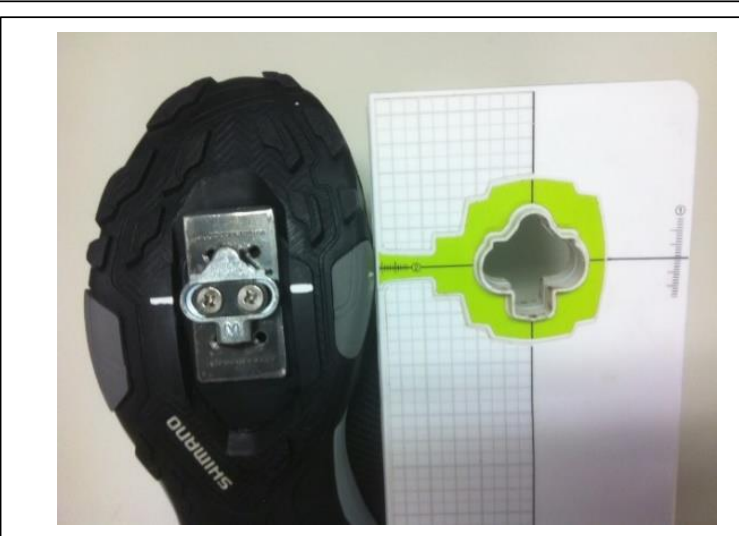

Figure 3: Custom-made system to increase the adjustment range of the cleats.

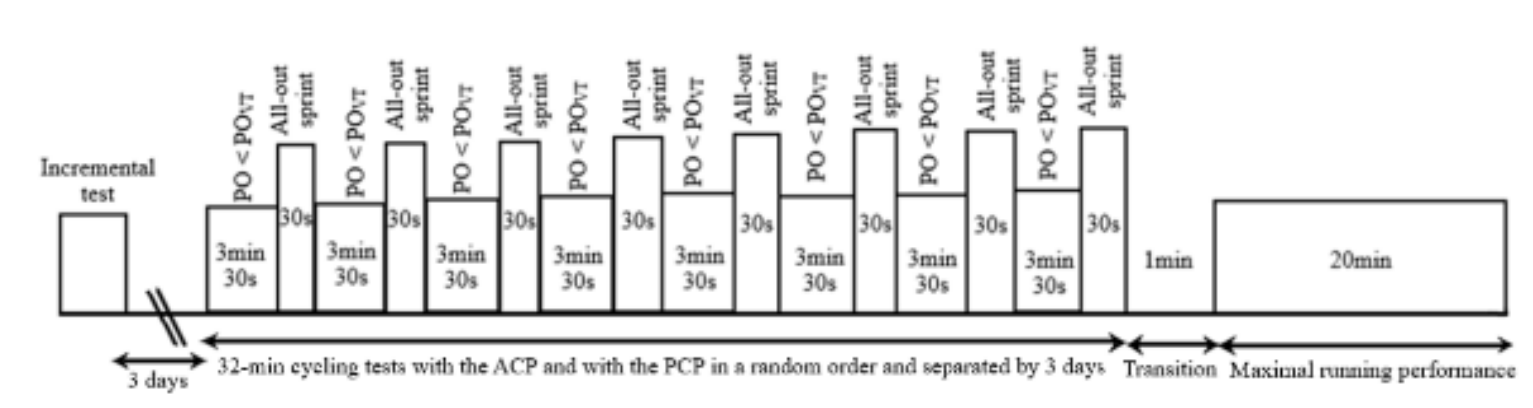

Figure 1: Cycle-run protocol. PCP: Posterior cleat position. ACP: Anterior cleat position. PO: Power output. POVT: Power output at first ventilatory threshold. 


\section{Measurements}

The PO and the pedalling cadence were measured with the cycle-ergometer during the graded maximal cycling test and during the experimental cycling tests. The reproducibility and the accuracy of the PO measurement using the Wattbike have been shown previously by Hopker et al. (2010) and Driller et al. (2014). The MAP was calculated according to the formula of Hopker et al. (2008):

$$
M A P(W)=W_{E}(W)+\left(\frac{20 W}{t(s)} \times t_{e}(s)\right)
$$

Where $\mathrm{MAP}=$ maximal aerobic power; $\mathrm{W}_{\mathrm{E}}=$ power output of last stage completed; $30 \mathrm{~W}=$ work rate increment; $\mathrm{t}=$ work rate duration; and $t_{e}=$ duration of the final stage.

During the experimental tests, the $\mathrm{PO}$ and the pedalling cadence were averaged during each sub-maximal interval of $3 \mathrm{~min} 30 \mathrm{~s}$ and sprint of $30 \mathrm{~s}$ and during the $32 \mathrm{~min}$ of the pedalling exercise. During the $\mathrm{TT}_{20}$, the distance covered by the participants and the speed were measured with the treadmill. The pacing strategy was then assessed by analysing the performance during the first 5-min portion of the $\mathrm{TT}_{20}$ and the overall performance during the $\mathrm{TT}_{20}$.

After a standard calibration of the gas analysis system following the manufacturer's instructions, gas exchanges (oxygen uptake: $\mathrm{VO}_{2}$; carbon dioxide production: $\mathrm{VCO}_{2}$ ) were collected continuously cycle to cycle during the graded maximal cycling test and the two experimental tests with a wireless and portable system (Oxycon Pro, Erich Jaeger GmbH, Hoechberg, Germany). This portable open-circuit indirect system allows for accurate measurements of physiological variables during high and low exercise intensities (Rietjens et al. 2001). The Oxycon Pro requires that participants wear a face mask and two small units mounted in a harness attached to the upper torso. The $\mathrm{VO}_{2}$ was averaged every $30 \mathrm{~s}$, and the $\mathrm{VO}_{2 \max }$ was defined as the highest $30 \mathrm{~s}$ average value of $\mathrm{VO}_{2}$ recorded during the graded maximal cycling test (Paton and Jardine 2012). During the experimental tests, the $\mathrm{VO}_{2}$ was averaged while the $32 \mathrm{~min}$ of the pedalling exercise as well as while the first 5 -min portion of the $\mathrm{TT}_{20}$ and the overall $\mathrm{TT}_{20}$. Heart rate (HR) was measured continuously during each test with a cardio transmitter (RCX3; Polar Electro Oy, Kempele, Finland).

The energy cost of running (EC) was calculated during the first 5 -min portion of the $\mathrm{TT}_{20}$ running section and during the overall $\mathrm{TT}_{20}$ following the equation of Di Prampero (1986):

$$
E C\left(m L O_{2} \cdot \mathrm{kg}^{-1} \cdot \mathrm{km}^{-1}\right)=\frac{\mathrm{VO}_{2}\left(\mathrm{mLO} \mathrm{O}_{2} \cdot \mathrm{kg}^{-1} \cdot \mathrm{min}^{-1}\right)}{\operatorname{Speed}\left(\mathrm{km} \cdot \mathrm{min}^{-1}\right)}
$$

The surface electromyographic (EMG) activity of the vastus lateralis (VL), vastus medialis (VM), biceps femoris (BF), gastrocnemius medialis $(\mathrm{GM})$, soleus (SOL), and tibialis anterior (TA) muscles of the right lower limb were measured continuously using adhesive circular electrodes (Ø: 10 mm; Ag/ AgCl BlueSensor N-00-S, Ambu A/S, Ballerup, Denmark), during the both the cycling exercise and the TT running of the two experimental tests. Two electrodes were spaced $20 \mathrm{~mm}$ apart, positioned on the centre of each muscle's belly, and aligned to the direction of the muscles fibres according to the Surface Electromyography for the Non-Invasive Assessment of Muscles (SENIAM) recommendations (Rainoldi et al. 2004). Three reference electrodes were placed on the anteroposterior iliac crest. The skin of the electrode attachment sites was shaved, slightly rubbed with an abrasive paste (Nuprep MLA1093, ADInstruments, Dunedin, New Zealand) and cleaned with alcohol. The EMG sensors were attached to the skin with a medical adhesive and were secured by the wearing of cycling leggings. The EMG signals were recorded with three synchronised 16-bit amplifier systems (PowerLab 26T; ADInstruments) at a sampling rate of 1000 $\mathrm{Hz}$, with a gain of 1000 and a common mode rejection $>80 \mathrm{~dB}$. All EMG signals were filtered with a band-pass filter $(10-450 \mathrm{~Hz})$ and were stored in LabChart software (version 7.0; ADInstruments). During analysis, the intensity of the muscle activity was quantified using the rootmean-square (RMS) of the EMG signal for the 
complete cycling test, for both the sub-maximal and the supra-maximal cycling intensities, for the first 5-min portion of the running test and for the overall running test. The RMS values were expressed as a percentage of the mean RMS value measured during the first sub-maximal interval in Zone 1. The muscle activity of the overall lower limb (LL) was then computed as the mean of muscle activity of all lower limb muscles.

\section{Statistical analysis}

The statistical analysis was carried out using Past software (version 3.18; Øyvind Hammer, Natural History Museum, University of Oslo, Norway). The data presented in the text, tables, and figures correspond to the mean values \pm SD and $95 \%$ confidence intervals $(95 \% \mathrm{CI})$ of the mean differences. The normality of the distribution and homogeneity of the variances were assessed with Shapiro-Wilk and Levene's tests, respectively. Student's t-tests, or pairwise Wilcoxon tests in the cases of non-homogeneity of the variances or non-normality of the distribution, were used to analyse the influence of the antero-posterior cleat position on the physiological (VO2, FC, and EC of running), biomechanical (muscle activity), and performance (Cycling PO, running speed, and running distance) variables during the overall cycling, sub-maximal, and supra-maximal cycling intensities, as well as during the first 5min portion of the TT20 and during the overall TT20 running test. The level of significance was set at $\mathrm{p}<0.05$. The effect sizes (ES) were calculated by the ratio of the mean to the SD in the difference of scores. As reported by Cohen (1992), we defined effects as small $(<0.2)$, medium $(0.2-$ $0.8)$ and large $(>0.8)$. We considered that a large effect corresponded to substantial changes (Cohen, 1988).

\section{Results}

The average $\mathrm{VO} 2 \mathrm{max}$, absolute $\mathrm{MAP}$, and relative MAP were $54.7 \pm 3.8 \mathrm{~mL} / \mathrm{min} / \mathrm{kg}$ (range: 49.5-59.2), $268 \pm 37 \mathrm{~W}$ (range: 194-325), and $4.43 \pm 0.39 \mathrm{~W} / \mathrm{kg}$ (range: 3.87-4.95), respectively. The average POVT was $161 \pm 22 \mathrm{~W}$ (range: 131-195), representing $61 \%$ of the MAP.

\section{Performance and physiological variables during cycling}

Figure 4 shows the evolution of the PO for one participant throughout the 32 min-cycling exercises performed with the PCP and with the $\mathrm{ACP}$. The mean PO during the $32 \mathrm{~min}$ of the cycling exercise for the seven triathletes was $180.6 \pm 26.8 \mathrm{~W}$ for the PCP and $190.0 \pm 20.2 \mathrm{~W}$ for the ACP ( $p=0.17$; ES $=0.40$; 95\% CI: $-3.0,21.8$ $\mathrm{W})$. The mean PO during the 8 intervals in Zone 1 was $151.9 \pm 21.5 \mathrm{~W}$ for the PCP and $161.1 \pm 13.4$ $\mathrm{W}$ for the ACP ( $\mathrm{p}=0.19$; $\mathrm{ES}=0.53$; 95\% CI: -3.5 , $21.9 \mathrm{~W})$. The mean PO during the 8 sprints in Zone 4 was $395.4 \pm 32.8 \mathrm{~W}$ for the PCP and 401.9 $\pm 30.7 \mathrm{~W}$ for the ACP ( $\mathrm{p}=0.55$; ES $=0.21 ; 95 \% \mathrm{CI}$ : -14.1, 26.9 W). We noticed a slightly better performance with the ACP compared to the PCP of $5.0 \%, 5.7 \%$, and $1.6 \%$ during the overall cycling exercise, the Zone 1 intervals, and the Zone 4 sprints, respectively, but none reached the level of significance, and the effects were small. In addition, the HR was similar between the PCP and ACP $(156 \pm 17 \mathrm{bpm}$ vs. $156 \pm 17$ bpm; $\mathrm{p}=0.98 ; \mathrm{ES}=0.01 ; 95 \% \mathrm{CI}:-3.8,3.7 \mathrm{bpm}$ ) and the $\mathrm{VO} 2$ was not significantly different between the two conditions of cleat placement $\left(33.9 \pm 1.7 \mathrm{mLO} 2 \cdot \mathrm{kg}^{-1} \cdot \mathrm{min}^{-1}\right.$ vs. $35.3 \pm 2.9$ $\mathrm{mLO} 2 \cdot \mathrm{kg}^{-1} \cdot \mathrm{min}^{-1}$, respectively; $\mathrm{p}=0.15 ; \mathrm{ES}=$ $0.61 ; 95 \%$ CI: $\left.0.1,3.0 \mathrm{mLO}^{2} \cdot \mathrm{kg}^{-1} \cdot \mathrm{min}^{-1}\right)$.

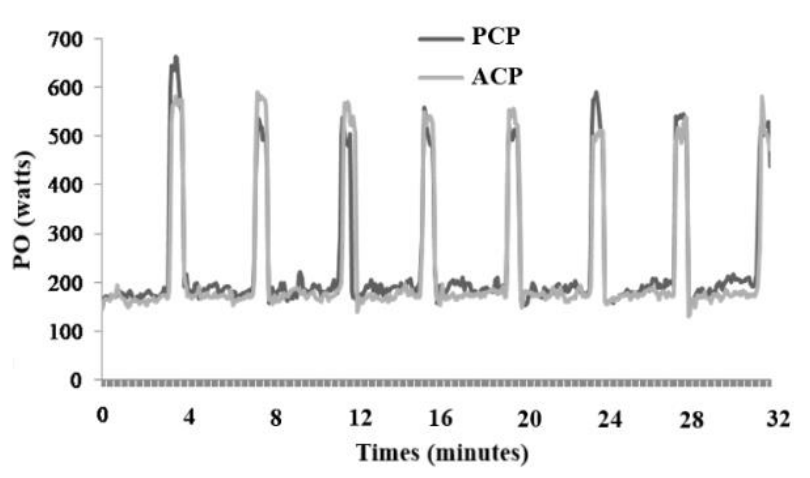

Figure 4: Evolution of crank power output (PO) throughout the 32-min cycling tests (including 8 sets of $3 \mathrm{~min} 30 \mathrm{~s}$ intervals performed at just below the power output at the first ventilatory threshold interspersed with $30 \mathrm{~s}$ sprints at $>100 \%$ of the maximal aerobic power) with the posterior cleat position (PCP) and with the anterior cleat position (ACP) for one participant. 


\section{Performance and physiological variables during running}

Table 1 shows the performance data and the physiological responses measured during the TT running test. The speed and the total running distance covered during the TT20 was very similar between the two experimental conditions (with a difference of $<0.2 \%$ ) even though the distance covered during the first 5min portion of the TT20 increased by $4.3 \%$ with the ACP (which did not reach the level of significance). Although we found no significant differences in the $\mathrm{HR}$ and $\mathrm{VO} 2$, we can observe that the HR increased $3.6 \%$ during the TT20 and the $\mathrm{VO} 2$ increased $4.9 \%$ during the first $5 \mathrm{~min}$ portion of the TT20 and by $6.5 \%$ during the overall TT20 with the ACP. The EC during the overall TT20 was $5.9 \%$ lower with the PCP (p = $0.04 ; \mathrm{ES}=0.37 ; 95 \%$ CI: $-1.8,-18.1 \%)$. No significant difference in the EC was found during the first 5-min portion of the TT20.

\section{Muscle activity}

Table 2 presents the normalised RMS values of the muscle activity measured with the PCP and with the ACP during the overall cycling exercise, the 8 intervals in Zone 1, and the 8 sprints in Zone 4. During the overall cycling test and the supra-maximal intensity, no significant difference was detected. However, during Zone 1 the SOL activity was reduced with the PCP ($7.0 \% ; \mathrm{p}=0.04 ; \mathrm{ES}=0.88 ; 95 \% \mathrm{CI}:-2.4,-12.6 \%$ ).

Table 3 presents the normalised RMS values of the muscle activity measured with the PCP and with the ACP during the first 5 min portion of the TT20 and the overall TT20. The GM was more activated with the ACP during the overall TT20 (+25\%; p $=0.04 ; \mathrm{ES}=1.05 ; 95 \%$ CI: 15.6, $95.5 \%)$ and during the first 5 min portion of the TT20 (+20\%; p = 0.10; ES = 0.95; 95\% CI: -0.38 , $84.5 \%)$. No other significant difference was detected.

Table 1: The mean (SD) of the running speed, running distance, heart rate (bpm), oxygen consumption (VO2), and energy cost (EC) during the first 5-min portion of the TT20 and the overall TT20.

\begin{tabular}{|c|c|c|c|c|c|c|c|c|}
\hline & \multicolumn{4}{|c|}{$1^{\text {st }} 5$-min of $\mathrm{TT}_{20}$} & \multicolumn{4}{|c|}{$\mathrm{TT}_{20}$} \\
\hline & PCP & $\mathrm{ACP}$ & $p$ value & ES & PCP & $\mathrm{ACP}$ & $p$ value & ES \\
\hline Speed $\left(\mathrm{km} \cdot \mathrm{h}^{-1}\right)$ & $13.5(0.7)$ & $14.1(1.0)$ & 0.40 & 0.71 & $14.6(0.6)$ & $14.6(0.9)$ & 0.89 & 0.04 \\
\hline Distance (m) & $1120(60)$ & $1170(83)$ & 0.40 & 0.70 & 4865 (200) & $4873(290)$ & 0.95 & 0.03 \\
\hline HR (bpm) & $171(10)$ & $170(10)$ & 0.85 & 0.10 & $167(15)$ & $173(9)$ & 0.19 & 0.50 \\
\hline $\mathrm{VO}_{2}\left(\%\right.$ of $\left.\mathrm{VO}_{2 \max }\right)$ & $65.6(7.5)$ & $69.0(10.9)$ & 0.29 & 0.37 & $70.8(11.3)$ & $75.7(9.6)$ & 0.26 & 0.47 \\
\hline $\mathrm{EC}\left(\mathrm{mLO}_{2} \cdot \mathrm{km}^{-1} \cdot \mathrm{kg}^{-1}\right)$ & $158.2(17.6)$ & $159.1(21.2)$ & 0.81 & 0.05 & $159.1(27.7)$ & $169.0(26.4)$ & 0.04 & 0.37 \\
\hline
\end{tabular}


Table 2: Mean (SD) of muscle activity of each lower body muscle during the submaximal intensity (Zone 1 intensity), the sprints (Zone 4 intensity) and the overall cycling test with the posterior cleat position (PCP) and the anterior cleat position (ACP). All RMS mean values are expressed in \% to the RMS value measured during the first cycling interval in Zone 1. ES: Effect size.

\section{Zone 1 intensity \\ Zone 4 intensity \\ Overall Cycling test}

\begin{tabular}{ccccccccccccc}
\hline & PCP & ACP & p & PCP & ACP & p & & PCP & ACP & p & ES \\
& $(\%)$ & $(\%)$ & value & & $(\%)$ & $(\%)$ & value & & (\%) & (\%) & value & \\
\hline VL & $110(9)$ & $108(13)$ & 1.00 & 0.18 & $208(49)$ & $187(51)$ & 0.08 & 0.42 & $131(13)$ & $125(19)$ & 0.30 & 0.38 \\
VM & $108(13)$ & $102(12)$ & 0.43 & 0.48 & $206(57)$ & $178(41)$ & 0.11 & 0.57 & $129(18)$ & $118(16)$ & 0.24 & 0.65 \\
BF & $90(21)$ & $97(17)$ & 0.46 & 0.37 & $212(61)$ & $190(59)$ & 0.08 & 0.37 & $121(16)$ & $119(24)$ & 0.81 & 0.10 \\
& & & & & & & & & & & & \\
TA & $86(20)$ & $84(9)$ & 0.81 & 0.14 & $183(60)$ & $153(40)$ & 0.07 & 0.60 & $107(20)$ & $100(12)$ & 0.27 & 0.44 \\
GM & $87(10)$ & $94(9)$ & 0.16 & 0.74 & $133(35)$ & $136(42)$ & 0.77 & 0.08 & $96(15)$ & $103(13)$ & 0.19 & 0.50 \\
& & & & & & & & & & & & \\
SOL & $93(8)$ & $100(8)$ & 0.04 & 0.88 & $170(23)$ & $155(27)$ & 0.08 & 0.60 & $110(12)$ & $111(15)$ & 0.59 & 0.07 \\
LL & $96(8)$ & $98(5)$ & 0.57 & 0.31 & $186(38)$ & $167(35)$ & 0.07 & 0.52 & $115(12)$ & $112(12)$ & 0.48 & 0.25 \\
\hline
\end{tabular}

VL: Vastus lateralis; VM: Vastus medialis; BF: Biceps femoris; TA: Tibialis anterior; GM: Gastrocnemius medialis; SOL: Soleus. The global activity of the lower limb (LL) was computed by the mean of activity of the 6 muscles. 
Table 3: Mean (SD) of muscle activity of each lower body muscle during the first 5-min portion of the TT20 and the overall TT20 with the posterior cleat position (PCP) and the anterior cleat position (ACP). All RMS mean values are expressed in \% to the RMS value measured during the first cycling interval in Zone 1. ES: Effect size.

\begin{tabular}{|c|c|c|c|c|c|c|c|c|}
\hline & \multicolumn{4}{|c|}{$1^{\text {st }} 5$-min of $\mathrm{TT}_{20}$} & \multicolumn{4}{|c|}{$\mathrm{TT}_{20}$} \\
\hline & $\begin{array}{l}\text { PCP } \\
(\%)\end{array}$ & $\begin{array}{l}\text { ACP } \\
(\%)\end{array}$ & $p$ value & ES & $\begin{array}{l}\text { PCP } \\
(\%)\end{array}$ & $\begin{array}{l}\text { ACP } \\
(\%)\end{array}$ & $P$ value & ES \\
\hline VL & $131(26)$ & $139(48)$ & 0.77 & 0.22 & $140(16)$ & $135(46)$ & 0.69 & 0.16 \\
\hline $\mathrm{VM}$ & $133(53)$ & $138(84)$ & 0.81 & 0.07 & $136(58)$ & $127(74)$ & 0.69 & 0.14 \\
\hline $\mathrm{BF}$ & $316(100)$ & $276(141)$ & 0.36 & 0.33 & $315(99)$ & $273(142)$ & 0.30 & 0.35 \\
\hline TA & $200(53)$ & $236(55)$ & 0.33 & 0.67 & $215(60)$ & $244(74)$ & 0.42 & 0.43 \\
\hline GM & $164(55)$ & 206 (33) & 0.10 & 0.95 & $165(53)$ & $220(52)$ & 0.04 & 1.05 \\
\hline SOL & $200(3)$ & $188(33)$ & 0.55 & 0.67 & $229(43)$ & $203(42)$ & 0.63 & 0.61 \\
\hline LL & $192(31)$ & 199 (51) & 0.59 & 0.17 & $200(41)$ & $201(50)$ & 0.93 & 0.02 \\
\hline
\end{tabular}

VL: Vastus lateralis; VM: Vastus medialis; BF: Biceps femoris; TA: Tibialis anterior; GM: Gastrocnemius medialis; SOL: Soleus. The global activity of the lower limb (LL) was computed by the mean of activity of the 6 muscles.

\section{Discussion}

The main results of this study were that modification of cleat position during the cycling part of a simulated Sprint triathlon influences the muscle activity and physiological responses during both the cycling and running parts of the race. The PCP improved the subsequent running economy without causing significant modifications in cycling power output. These physiological changes were associated with a reduction in the recruitment of the SOL during the sub-maximal cycling and of the GM during the subsequent running.

\section{Cycling test}

In the current study, the VO2 and power output were not significantly different between the PCP

and ACP. Several studies have focused on optimizing the cycling cleat position to reduce the risk of overuse injury (e.g. Ruby et al. 1992; Ruby and Hull 1993; Callaghan 2005) and to improve performance (Van Sickle and Hull 2007; Paton 2009). The antero-posterior cleat position has been considered as a critical factor for pedal force effectiveness (Grappe 2009). Hennig and Sanderson (1995) identified the first metatarsal head and the hallux as the major forcecontributing structures of the foot. Therefore, Grappe (2009) and Silberman et al. (2005) suggested that aligning the first metatarsal head 
with the centre of the cleat could improve cycling performance. Recently, authors have advocated positioning the centre of the cleat in alignment with the middle of the first and fifth metatarsal head for better stability of the foot (i.e. about $5 \mathrm{~mm}$ behind the first metatarsal) (FitzGibbon et al. 2016; Burt 2014). Several studies evaluated the effect of antero-posterior cleat placement on physiological variables in submaximal condition without observing any changes (Van Sickle and Hull 2007; Paton 2009; Paton and Jardine 2012; Viker and Richardson 2013). This is in agreement with our results despite of the fact that the current study simulated both sub-maximal and supramaximal conditions and that the PCP conditions in the previous studies corresponded to a very large posterior cleat displacement (not representative of the possible adjustments of cycling shoes currently available on the market).

In regard to muscle activity, SOL was less recruited with PCP during submaximal intensity but no difference was found for the other muscles. Litzenberger et al. (2008) and McDaniel et al. (2013) demonstrated that a mid-foot cleat placement led to a reduction in the GM and SOL activity during sub-maximal cycling. These two studies suggested that a lower recruitment of the calf-muscles could be caused by a decrease of the joint-torque by reducing the lever arm of the foot (pedal axis to ankle joint). However, we found no difference in SOL activity between ACP and PCP during the whole cycling test and the allout sprints. In addition, McDaniel et al. (2013) have shown that an aft cleat position induced a higher activity of the TA, VL, and gluteus maximus. Moreover, Litzenberger et al. (2008) have found a lower activity of the TA during the first part of the downstroke (between $0^{\circ}$ to $40^{\circ}$ of the pedalling cycle) when the cleat centre was aligned with the first metatarsal head compared to a mid-foot cleat position. Another study reported an increase in the TA activity between $0^{\circ}$ and $90^{\circ}, 180^{\circ}$ and $270^{\circ}$, and $270^{\circ}$ and $360^{\circ}$ of the pedalling cycle and in the rectus femoris activity between $270^{\circ}$ and $360^{\circ}$ of the pedalling cycle when using a $15 \mathrm{~mm}$ backward first metatarsal cleat position compared to a $15 \mathrm{~mm}$ forward first metatarsal cleat position (Chartogne et al. 2016). The present study showed a lower recruitment of the TA and of the VL with the ACP but the significant level was not reached, probably due to the small sample size.

\section{Running test}

During the subsequent TT20 running test, the EC was significantly lower after the cycling test performed with the PCP. However, no significant difference of performance (distance and speed), $\mathrm{HR}$, and $\mathrm{VO} 2$ was found during the overall running test. Previous study reported the advantage of using a posterior cleat placement for a subsequent $5.5 \mathrm{~km}$ treadmill run (Paton and Jardine 2012). However, Viker and Richardson (2013) found no benefit from using a posterior cleat position for draft-legal triathlon performance, which is consistent with our results. In addition, these two previous studies did not show modification of the $\mathrm{VO} 2$ between the two conditions of cleat placement, which is in accordance with the current study. Nonetheless, the EC of running improved after the cycling test performed with the PCP, which suggest that this condition would be favourable for saving energy during the running part. The improvement in EC could be linked to the lower SOL recruitment during the sub-maximal cycling test performed with PCP. This assumption supports the hypothesis of several previous studies concerning the improvement of subsequent running performance which may be associated with reduction in plantar flexor muscle activity during the cycling phase of the event (Litzenberger et al. 2008; Illes et al. 2010; Paton and Jardine 2012). However, the distance covered by the participants in the present study with the PCP and with the ACP were similar. This is probably due to the short time of the running portion (20 $\mathrm{min})$ because $\mathrm{EC}$ is considered as an important correlate of endurance running performance (Morgan et al. 1989). Therefore, despite the absence of significant difference in running distance, we can hypothesise that the PCP condition could increase performance during a triathlon with running parts longer than $5 \mathrm{~km}$ (i.e. Olympic, 
Half-Ironman, and Ironman), corroborating the conclusion of the Paton and Jardine study (2012).

The analysis of the first 5 min portion of the TT20 showed a faster start with the ACP. Moreover, we can note that the $\mathrm{VO} 2$ increased by almost $5 \%$ with ACP. Although these results were not significant, they could explain the higher EC measured during the TT20 running test in this condition. Viker and Richardson (2013) also observed that the first part of the 20 min running test was significantly slower with the PCP even if the overall performance was similar. We can observe a same tendency in our study but not to the level of significance, probably due to the smaller sample of participants. Pacing strategy, which is particularly influenced by the succession of the three disciplines of a triathlon, is considered as one of the most important factors for running performance (Hausswirth et al. 1996). It has been shown that a faster running start leads to a weaker overall running performance during a triathlon due to alterations in physiological variables (metabolic and ventilatory) (Hausswirth et al. 2010; Le Meur et al. 2011). Therefore, we can suggest that the changes in pacing strategy observed in the current study could explain the improvement in EC measured after the cycling test performed with PCP.

In regard to muscle activity, we have observed a lower recruitment of the GM during the TT20 and the first 5-min portion of the TT20 with the PCP. Thus, the running biomechanics may have deteriorated less as described by Illes et al. (2010), who found that the plantar pressure and the stride frequency are less altered after cycling using a posterior cleat position. Therefore, we can hypothesise that the decrease in muscle recruitment after the cycling test performed with the PCP associated with the changes in pacing strategy could be responsible for the improvement in EC during the running phase. Consequently, the current study shows the real impact of cleat positioning on muscle activity and performance-related variables for subsequent running.
In conclusion, this study suggests that the foreaft cleat position plays an important role in the biomechanical and physiological responses during a Sprint triathlon. The PCP seems to be more suitable to improve the subsequent running economy while decreasing the SOL activity during sub-maximal cycling and the GM activity during the subsequent running part. However, it is important to note that the SOL was recruited less only during the submaximal intensity. Future research should confirm these results with a larger sample of high-level adult competitors. Indeed, we can assume that the mixed sample in terms of age and gender was not optimal for this demanding protocol. In addition, the laboratory conditions require performing a time trial exercise for testing, which is not representative of race conditions and, in particular, the interaction with other triathletes. However, the integration of all-out sprints in the protocol seems more representative of Sprint triathlon competitions with large intensity variations. Moreover, our experimental conditions (PCP and $\mathrm{ACP}$ ) were different from those of previous studies (e.g. 1st metatarsal and mid-foot) which makes comparisons difficult. Therefore, it seems necessary to continue research to identify how the different cleat positioning configurations (e.g. ACP, 1st metatarsal, PCP, mid-foot) influences muscle activity and impacts performance-related variables by isolating different exercise intensities (e.g. sub-maximal, MAP, supra-maximal). Nevertheless, the present study provides original findings illuminating shoe cleat pedal interface optimisation and could help triathletes to determine their optimal cycling cleat adjustment.

\section{Practical applications}

This study has practical applications for triathletes who wish to improve their performance by optimising adjustments at the shoe-cleat-pedal interface. The PCP seems more beneficial for subsequent running and could be recommended for use in triathlons, especially if the cycling part is performed at a constant PO 
and/or the distance of the running part is greater than $5 \mathrm{~km}$.

\section{Acknowledgements}

The authors thank the Morphologics Company, particularly Michel Le Goallec, William Le Goallec and Martin Bouedo, for their involvement in this study.

\section{Conflict of interest}

No potential conflict of interest was reported by the authors.

\section{References}

1. Amann M, Subudhi A, Foster C (2004) Influence of testing protocol on ventilatory thresholds and cycling performance. Medicine \& Science in Sports \& Exercise $36 \quad$ (4):613-622. doi:10.1249/01.MSS.0000122076.21804.10

2. Bernard T, Vercruyssen F, Grego F, Hausswirth C, Lepers R, Vallier JM, Brisswalter J (2003) Effect of cycling cadence on subsequent $3 \mathrm{~km}$ running performance in well trained triathletes. British Journal of Sports Medicine 37 (2):154-159. doi:http://dx.doi.org/10.1136/bjsm.37.2.154

3. Bernard T, Vercruyssen F, Mazure C, Gorce P, Hausswirth C, Brisswalter J (2007) Constant versus variable-intensity during cycling: effects on subsequent running performance. European Journal of Applied Physiology 99 (2):103-111. doi:https:/ /doi.org/10.1007/s00421-006-0321-7

4. Bernard T, Hausswirth C, Le Meur Y, Bignet F, Dorel S, Brisswalter J (2009) Distribution of power output during the cycling stage of a triathlon world cup. Medicine \& Science in Sports \& $\quad$ Exercise $41 \quad$ (6):1296-1302. doi:10.1249/MSS.0b013e318195a233

5. Burt P (2014) Bike Fit: Optimise your bike position for high performance and injury avoidance. Bloomsbury Plublishing Plc Edition.

6. Callaghan MJ (2005) Lower body problems and injury in cycling. Journal of Bodywork and Movement Therapies 9 (3):226-236. doi:https://doi.org/10.1016/j.jbmt.2005.01.007
7. Chartogne M, Duc S, Bertucci W, Rodríguez JA, Pernía R (2016) Effect of shoes cleat position on physiological and biomechanical variables of cycling performance. Journal of Science and Cycling 5(2).

8. Cohen J (1988) Statistical power analysis for the behavioral sciences. Routledge Academic, Hillsdale, New Jersey.

9. Cohen J (1992) A power primer. Psychological bulletin, 112 (1):155-159.

10. De Vey Mestdagh K (1998) Personal perspective: in search of an optimum cycling posture. Applied Ergonomics $29 \quad$ (5):325-334. doi:https://doi.org/10.1016/S00036870(97)00080-X

11. Di Prampero PE (1986) Energy cost of human locomotion on land and in water. International Journal of Sports Medicine 7 (2):55-72.

12. Driller MW, Argus CK, Bartram JC, Bonaventura J, Martin DT, West NP, Halson SL (2014) Reliability of a 2-bout exercise test on a wattbike cycle ergometer. International Journal of Sports Physiology and Performance 9 (2):340-345. doi:https://doi.org/10.1123/ijspp.2013-0103

13. Dorel S, Guilhem G, Couturier A, Hug F (2012) Adjustment of muscle coordination during an allout sprint cycling task. Medicine \& Science in Sports \& Exercise $44 \quad$ (11):2154-2164. doi:10.1249/MSS.0b013e3182625423.

14. Etxebarria N, D'Auria S, Anson JM, Pyne DB, Ferguson RA (2014) Variability in power output during cycling in international Olympic-distance triathlon. International journal of sports physiology and performance 9 (4):732-734. doi:https:/ / doi.org/10.1123/ijspp.2013-0303

15. FitzGibbon S, Vicenzino B, Sisto SA (2016) Intervention at the foot-shoe-pedal interface in competitive cyclists. International Journal of Sports Physical Therapy 11(4):637-650.

16. Garside I, Doran DA (2000) Effects of bicycle frame ergonomics on triathlon $10-\mathrm{km}$ running performance. Journal of Sports Sciences 18(10):825-833.

doi:https:/ /doi.org/10.1080/026404100419883.

17. Grappe F (2009) Cyclisme et optimisation de la performance: science et méthodologie de l'entraînement. Bruxelles: de Boeck Université. 
18. Harriss DJ, Atkinson G (2011) Update-ethical standards in sport and exercise science research. International Journal of Sports Medicine 32 (11):819-821. doi:10.1055/s-0031-1287829

19. Hausswirth $C$, Bigard $A X$, Berthelot $M$, Thomaidis M, Guezennec CY (1996) Variability in energy cost of running at the end of a triathlon and a marathon. International Journal of Sports Medicine 17 (8):572-579. doi:10.1055/s-2007972897

20. Hausswirth C, Lehénaff D, Dréano P, Savonen K (1999) Effects of cycling alone or in a sheltered position on subsequent running performance during a triathlon. Medicine and Science in Sports and Exercise 31 (4):599-604. doi:10.1097/00005768-199904000-00018

21. Hausswirth C, Vallier JM, Lehenaff $D$, Brisswalter J, Smith D, Millet G, Dréano P (2001) Effect of two drafting modalities in cycling on running performance. Medicine and Science in Sports and Exercise 33 (3):485-492. doi:10.1097/00005768-200103000-00023

22. Hausswirth C, Brisswalter J (2008) Strategies for improving performance in long duration events. Sports Medicine $38 \quad$ (11):881-891. doi:10.2165/00007256-200838110-00001

23. Hausswirth C, Le Meur Y, Bieuzen F, Brisswalter J, Bernard T (2010) Pacing strategy during the initial phase of the run in triathlon: influence on overall performance. European Journal of

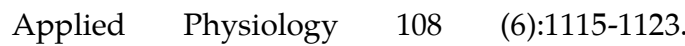
doi:10.1007/s00421-009-1322-0

24. Hennig EM, Sanderson DJ (1995) In-shoe pressure distributions for cycling with two types of footwear at different mechanical loads. Journal of Applied Biomechanics 11 (1):68-80. doi:https://doi.org/10.1123/jab.11.1.68

25. Hopker J, Coleman D, Passfield L (2009) Changes in cycling efficiency during a competitive season. Medicine Science in Sports Exercise 41 (4):912919. doi:10.1249/MSS.0b013e31818f2ab2

26. Hopker J, Myers S, Jobson SA, Bruce W, Passfield L (2010) Validity and reliability of the Wattbike cycle ergometer. International journal of sports medicine 31 (10):731-736. doi:10.1055/s-00301261968
27. Illes $S$, Hassmann $M$, Martin $H$, Ilagan $R$, Litzenberger S, Sabo A (2010) Influence of pedaling with the metatarsus on running characteristics in triathlon competition. Procedia

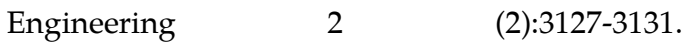
doi:https://doi.org/10.1016/j.proeng.2010.04.12 2

28. Jones AM, Doust JH (1996) A 1\% treadmill grade most accurately reflects the energetic cost of outdoor running. Journal of Sports Sciences 14 (4):321-327. doi:https://doi.org/10.1080/02640419608727717

29. Le Meur $Y$, Bernard T, Dorel S, Abbiss CR, Honnorat G, Brisswalter J, Hausswirth C (2011) Relationships between triathlon performance and pacing strategy during the run in an international competition. International Journal of Sports Physiology and Performance 6 (2):183194. doi:https://doi.org/10.1123/ijspp.6.2.183

30. Litzenberger S, Illes S, Hren M, Reichel M, Sabo A (2008) Influence of Pedal Foot Position on Muscular Activity during Ergometer Cycling. In: The Engineering of Sport $7: 215-222$.

31. McDaniel TM, Fu YC, Simpson KJ (2013) The effects of cleat location on muscle recruitment strategies of cycling. In ISBS-Conference Proceedings Archive (Vol. 1, No. 1).

32. Morgan DW, Martin PE, Krahenbuhl GS (1989) Factors affecting running economy. Sports Medicine 7 (5):310-330. doi:10.2165/00007256198907050-00003

33. Paavolainen L, Nummela A, Rusko H, Häkkinen K (1999) Neuromuscular characteristics and fatigue during $10 \mathrm{~km}$ running. International journal of sports medicine 20 (08):516-521. doi:10.1055/s-1999-8837

34. Paton CD (2009) Effects of shoe cleat position on physiology and performance of competitive cyclists. International Journal of Sports Physiology and Performance 4 (4):517-523. doi:https:/ /doi.org/10.1123/ijspp.4.4.517

35. Paton CD, Jardine T (2012) The effects of cycling cleat position on subsequent running performance in a simulated duathlon. Journal of Science and Cycling 1 (1):15-20. 
36. Rainoldi A, Melchiorri G, Caruso I (2004) A method for positioning electrodes during surface EMG recordings in lower limb muscles. Journal of Neuroscience Methods 134 (1):37-43. doi:https://doi.org/10.1016/j.jneumeth.2003.10. 014

37. Rietjens GJWM, Kuipers H, Kester ADM, Keizer HA (2001) Validation of a computerized metabolic measurement system (Oxycon-Pro ${ }^{\circledR}$ ) during low and high intensity exercise. International Journal of Sports Medicine 22 (04):291-294. doi:10.1055/s-2001-14342

38. Ruby P, Hull ML, Kirby KA, Jenkins DW (1992) The effect of lower-limb anatomy on knee loads during seated cycling. Journal of Biomechanics 25 (10):1195-1207.

doi:https:/ / doi.org/10.1016/00219290(92)90075-C

39. Ruby P, Hull ML (1993) Response of intersegmental knee loads to foot/pedal platform degrees of freedom in cycling. Journal of Biomechanics $26 \quad$ (11):1327-1340. doi:https:/ /doi.org/10.1016/00219290(93)90356-J

40. Silberman MR, Webner D, Collina S, Shiple BJ (2005) Road bicycle fit. Clinical Journal of Sport Medicine 15

(4):271-276. doi:10.1097/01.jsm.0000171255.70156.da

41. Van Sickle JR, Hull ML (2007) Is economy of competitive cyclists affected by the anteriorposterior foot position on the pedal? Journal of $\begin{array}{lll}\text { Biomechanics } & 40 & (6): 1262-1267 .\end{array}$ doi:https://doi.org/10.1016/j.jbiomech.2006.05. 026

42. Viker T, Richardson MX (2013) Shoe cleat position during cycling and its effect on subsequent running performance in triathletes. Journal of Sports Science 31 (9):1007-1014. doi:https:/ /doi.org/10.1080/02640414.2012.7607 48

43. Wu SSX, Peiffer JJ, Brisswalter J, NosakaK, Lau WY, Abbiss CR (2015) Pacing strategies during the swim, cycle and run disciplines of sprint, Olympic and half-Ironman triathlons. European Journal of Applied Physiology 115 (5):1147-1154. doi:https:/ /doi.org/10.1007/s00421-014-3096-2 